\title{
A INDEPENDÊNCIA FUNCIONAL E A MANUTENÇÃO DOS PAPÉIS OCUPACIONAIS EM SUJEITOS COM SEQUELAS NEUROLÓGICAS
}

\author{
Elaine Janeczko Navarro', Diane Priscila Stoffel ${ }^{2}$, Renato Nickel ${ }^{3}$
}

\begin{abstract}
RESUMO: Estudo descritivo e transversal objetivou verificar possíveis relações entre nível de independência funcional, perda, manutenção e/ou resgate de papéis ocupacionais e fatores pessoais de 46 pessoas sequeladas por doenças neurológicas. A pesquisa foi aprovada por comitê de ética e os dados foram coletados por meio da Medida de Independência Funcional, da Lista de Identificação de Papéis Ocupacionais e leitura dos prontuários. Os papéis de serviço doméstico, membro de família e passatempo/amador apresentaram maiores índices de desempenho contínuo. Observou-se alta frequência de aposentadoria em idade produtiva, embora os participantes tenham designado muita importância ao papel de trabalhador e apresentarem nível de independência compatível com a possibilidade de desempenho desse papel. Ressalta-se a hipótese de que a doença e a deficiência podem não ser os principais fatores determinantes de limitações na atividade e restrições na participação de pessoas sequeladas, enfatizando a influência de fatores pessoais e ambientais nesses aspectos.

DESCRITORES: Terapia ocupacional; Classificação internacional de funcionalidade, incapacidade e saúde; Pessoas com deficiência; Comportamento; Atividades cotidianas.

\section{FUNCTIONAL INDEPENDENCE AND MAINTENANCE OF OCCUPATIONAL ROLES IN SUBJECTS WITH NEUROLOGICAL SEQUELAE}

\begin{abstract}
This descriptive and cross-sectional study aimed to verify possible relations between level of functional independence, loss, maintenance and/or recovery of occupational roles, and personal factors of 46 persons with neurological sequelae. The research was approved by the ethics committee and the data was collected through the Measurement of Functional Independence, the List of Identification of Occupational Roles and reading of the hospital records. The roles of domestic service, family member and pass-time/amateur present the highest rates of continuous performance. A high frequency of retirement at a productive age was observed, although the participants assign much importance to the role of worker and present a level of independence which is compatible with the possibility of performance of this role. The hypothesis that illness and disability may not be the main determinant factors for limitations on activity and restrictions on the participation of persons with sequelae is stressed, emphasizing the influence of personal and environmental factors on these aspects. DESCRIPTORS: Occupational therapy; International classification of functionality, disability and health; Persons with disability; Behavior; Routine activities.
\end{abstract}

\section{LA INDEPENDENCIA FUNCIONAL Y LA MANUTENCIÓN DE LOS PAPELES OCUPACIONALES EN SUJETOS CON SECUELAS NEUROLÓGICAS}

RESUMEN: Estudio descriptivo y transversal cuya finalidad fue verificar posibles relaciones entre nivel de independencia funcional, pérdida, manutención y/o rescate de papeles ocupacionales y factores personales de 46 personas con secuelas por enfermedades neurológicas. La investigación fue aprobada por comité de ética y los datos fueron obtenidos por medio de la Medida de Independencia Funcional, de la Lista de Identificación de Papeles Ocupacionales y lectura de los prontuarios. Los papeles de servicio doméstico, miembro de familia y pasatiempo/hobby presentaron mayores índices de desempeño continuo. Se observó gran frecuencia de jubilación en edad productiva, a pesar de que los participantes tengan designado mucha importancia al papel del trabajador y presentar nivel de independencia compatible con la posibilidad de desempeño de ese papel. Se destaca que la hipótesis de que la enfermedad y la deficiencia pueden no ser los principales factores determinantes de limitaciones en la actividad y restricciones en la participación de personas secueladas, enfatizando la influencia de factores personales y ambientales en eses aspectos. DESCRIPTORES: Terapia ocupacional; Clasificación internacional de funcionalidad, incapacidad y salud; Personas con deficiencia; Comportamiento; Actividades cotidianas.

${ }^{1}$ Terapeuta Ocupacional. Membro do Grupo de Pesquisa de Terapia Ocupacional da Universidade Federal do Paraná -UFPR.

${ }^{2}$ Terapeura Ocupacional. Especializanda em Reabilitação Neurofuncional pela Faculdade Inspirar. Membro do Grupo de Pesquisa de Terapia Ocupacional da UFPR.

${ }^{3}$ Terapeuta Ocupacional. Doutor em Educação. Professor do Curso de Graduação em Terapia Ocupacional da UFPR.

Autor correspondente:

Renato Nickel

Universidade Federal do Paraná

Rua Lothário Meissner, 632 - 80210-170 - Curitiba-PR-Brasil

E-mail: rnickel@ufpr.br
Recebido: 04/03/2013 Aprovado: 11/09/2013 


\section{INTRODUÇÃO}

Atualmente observa-se aumento das sequelas decorrentes das disfunções neurológicas em adultos devido ao envelhecimento populacional, à maior exposição aos fatores de risco e aos avanços da medicina para manutenção da vida ${ }^{(1)}$. Contudo, esta vida mantida nem sempre está livre de efeitos adversos, gerando às pessoas sequeladas, e suas famílias, problemas de ordem física, psicológica, social e econômica ${ }^{(2-3)}$. Dados do censo brasileiro de 2010 apontam que $23,90 \%$ da população tem algum tipo de deficiência, e que deste total, $92,67 \%$ dizem respeito a pessoas acima de 15 anos de idade ${ }^{(4)}$.

No âmbito da reabilitação, observa-se que pacientes recuperados fisicamente, ou com sequelas leves, nem sempre apresentam como resultado a independência para o desempenho nas atividades do dia-a-dia e, menos ainda, um comportamento de retorno aos seus papéis ocupacionais/sociais. Entende-se por papéis ocupacionais ações produtivas que organizam o comportamento e a rotina diária de uma pessoa, sendo que exercê-lo, depende de uma organização complexa, que se estabelece pelo convívio da pessoa em sociedade, o que permite, com o passar do tempo, a internalização destes papéis por meio de atitudes e comportamentos que correspondem às expectativas sociais ${ }^{(5)}$.

Justificado por estes fatores, esta pesquisa tem por objetivo verificar possíveis relações entre o nível de independência funcional e papéis ocupacionais (perda, manutenção e/ou resgate) de sujeitos acometidos por sequelas neurológicas. O tema em questão é relacionado à saúde da pessoa com deficiência, suas atividades cotidianas e retorno ao trabalho.

\section{MÉTODO}

Esta é uma pesquisa descritiva, transversal, de amostra por conveniência. Foram incluídos pessoas com sequelas neurológicas, idade superior a 18 anos, de ambos os gêneros, atendidos no Ambulatório de Espasticidade de um hospital de ensino da capital paranaense entre março e setembro de 2009. Apresentar comprometimento cognitivo foi considerado critério de exclusão, ou seja, foram eliminados do estudo aqueles com pontuação abaixo de 6 na parte cognitiva da Medida de Independência Funcional (MIF). O recrutamento dos participantes foi realizado de acordo com o fluxo de atendimento no referido ambulatório. Os dados foram coletados por meio de aplicação de entrevista e avaliação em sala específica, após a leitura e a assina- tura do Termo de Consentimento Livre e Esclarecido.

A avaliação quantitativa de cuidados necessários para a realização de tarefas diárias, motoras e cognitivas foi realizada por meio da MIF e os aspectos relacionados às atividades cotidianas, de trabalho e socialização - papéis ocupacionais - foram identificados por meio da Lista de Identificação de Papéis Ocupacionais. Ambas as avaliações foram validadas no Brasil( ${ }^{(6-7)}$.

O objetivo principal da MIF é avaliar, de maneira quantitativa, a carga de cuidados que um indivíduo com restrições funcionais, de origem variada, necessita para realizar determinadas atividades da vida diária. Tal avaliação é composta por 18 itens, sendo que 13 dizem respeito ao desempenho motor nessas atividades e cinco correspondem a questões cognitivas, as quais foram consideradas no critério de inclusão e exclusão. A pontuação em cada item varia de 1 a 7 -progressivamente de dependência completa à independência total-, com critérios previamente estabelecidos para essa pontuação em cada atividade. $\mathrm{O}$ escore final é compreendido em um intervalo de 18 a 126 pontos $^{(6)}$. A pontuação em questão foi dividida, conforme validação, em três níveis: dependência completa (18 a 45), dependência moderada (46 a 99) e independência completa (100 a 126).

A Lista de Identificação de Papéis Ocupacionais é utilizada para identificar essas ações produtivas de uma pessoa ao longo da vida. A lista compreende os papéis de estudante, trabalhador, voluntário, cuidador, serviço doméstico, amigo, membro de família, religioso, passatempo/amador, participante em organizações e outros, e é constituída por duas partes: uma relativa ao desempenho percebido dos papéis ocupacionais pelos sujeitos, e outra que envolve a importância designada pelo sujeito a cada papel ocupacional. Desse modo, os papéis ocupacionais são caracterizados pelo sujeito conforme seu desempenho no passado, presente e futuro, e em grau de nenhuma, alguma ou muita importância, proporcionando, assim, a informação da condição do sujeito em equilibrar e manter seus papéis ocupacionais ${ }^{(7)}$.

Os dados levantados por meio da MIF, da Lista de Identificação de Papéis Ocupacionais e fatores pessoais foram categorizados conforme as características das variáveis, para posteriormente serem descritos em sua frequência e analisados em possíveis relações apresentadas em tabelas cruzadas (estatística descritiva).

Este estudo segue a resolução 196/96 CONEP e foi aprovado pelo Comitê de Ética, como parte do projeto de pesquisa "Estudo descritivo da relação entre a qualidade de vida e o nível de independência funcional do sujeito com sequelas neurológicas e de seu cuidador", sob o registro 1524.189/2007-09. 


\section{RESULTADOS}

Foram coletados dados de 46 participantes, com média de idade de 45,28 anos $( \pm 14,39)$, sendo $65,2 \%$ do gênero feminino, $45,7 \%$ casados, $52,2 \%$ com ensino fundamental incompleto e $54,3 \%$ aposentados.

Dentre os diagnósticos médicos destacou-se, primeiramente, o acidente vascular cerebral (AVC) com $36,9 \%$; seguido pela paralisia cerebral (PC) com $15,2 \%$; paraparesia espástica com $13 \%$; e esclerose múltipla (EM), com 10,8\%. Os demais diagnósticos encontrados foram distonia generalizada com 6,5\%; mielite transversa, lesão medular e cãibra do escrivão com $4,3 \%$ cada; e a espondilite anquilosante e o traumatismo crânio encefálico (TCE) com $2,1 \%$ cada.

Os resultados da MIF foram divididos, como informado, em três níveis de independência/dependência funcional, sendo que nenhum participante apresentou dependência completa (nível 1), 10(21,7\%) apresentaram dependência moderada (nível 2) e 36(78,3\%) apresentaram independência completa (nível 3). O menor escore da MIF total foi de 48 e o maior de 126; a média da MIF total foi de 107,22 ( $( \pm 16,7)$ e a moda 117.

Os resultados obtidos por meio da Lista de
Identificação de Papéis Ocupacionais em relação ao desempenho percebido estão apresentados no Gráfico 1. Com base nesses dados, ressalta-se que o papel de serviço doméstico apresentou o índice mais elevado de desempenho contínuo, sem rompimento $(80,4 \%)$, seguido pelo papel de membro de família $(78,3 \%)$ e passatempo/amador (73,9\%). O papel de estudante foi caracterizado predominantemente pelo desempenho somente no passado $(58,7 \%)$, seguido da perda somente no presente $(28,3 \%)$. Por outro lado, $50 \%$ dos participantes tem perspectiva de assumir o papel de voluntário no futuro.

No papel de trabalhador, destacou-se a questão da perda somente no presente $(39,1 \%)$, seguido do desempenho apenas no passado (23,9\%).

Os papéis desempenhados até o presente apresentaram índices abaixo de 5\%, indicando uma ruptura nos papéis ocupacionais.

Observa-se no Gráfico 2, referente à importância designada aos papéis ocupacionais, a existência de alto grau de importância informados pelos participantes, apesar dos índices de desempenho ocupacionais indicarem descontinuidade dos mesmos.

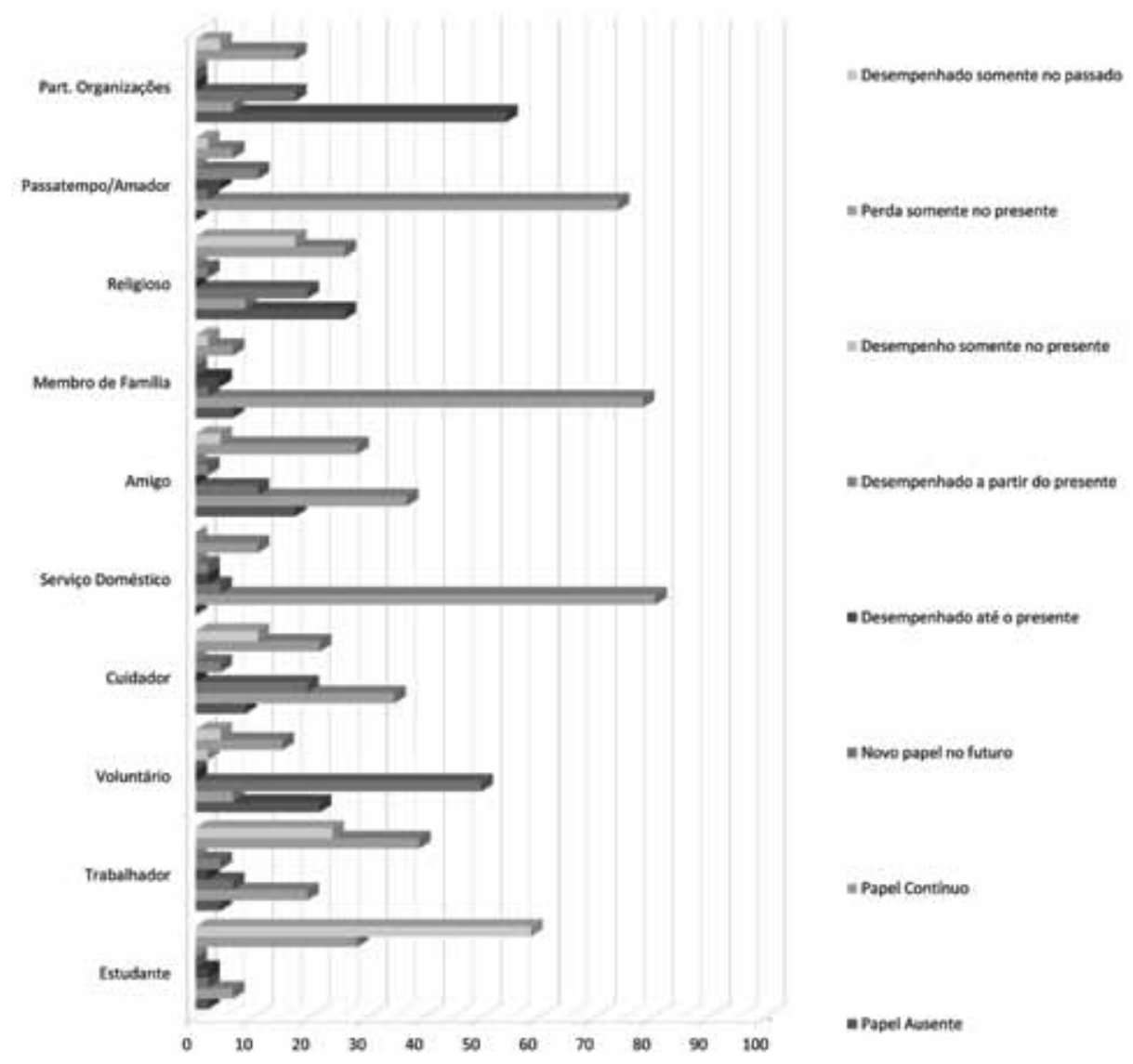

Gráfico 1 - Padrão de desempenho de papéis ocupacionais. Curitiba, 2009 


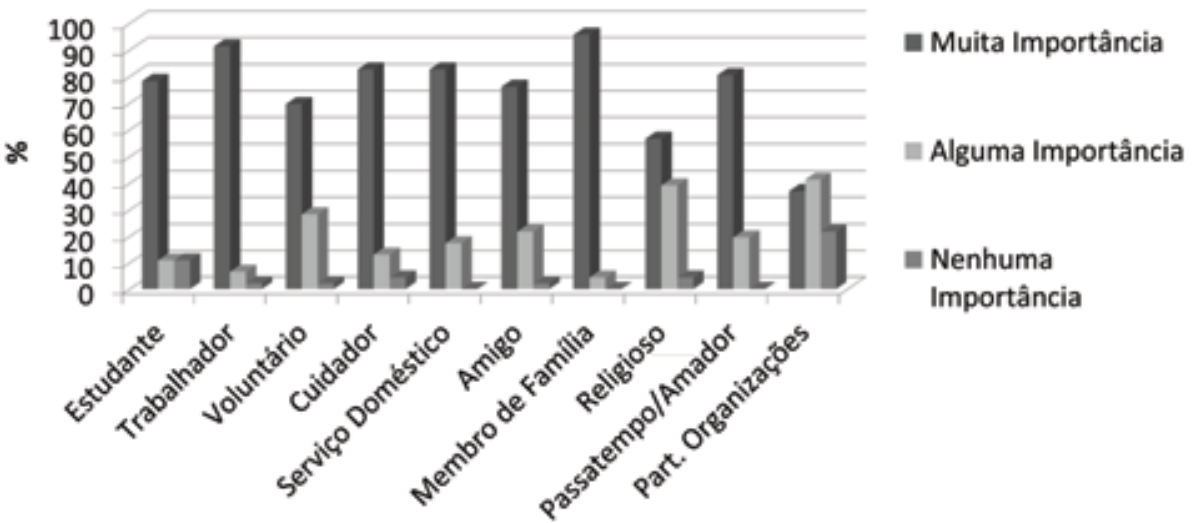

Gráfico 2 - Importância designada aos papéis ocupacionais. Curitiba, 2009

Em divisão por gênero, na relação entre faixa etária e ocupação atual, tem-se que $54,1 \%$ das mulheres entre 18 e 59 anos estão aposentadas, mesmo sendo esta faixa etária considerada produtiva. Outro aspecto relevante é que $95,8 \%$ dessas mulheres apresentaram nível de independência completa na MIF, com média de $112,17( \pm 10,82)$ e moda de 117 .

Em relação aos homens, na faixa etária de produtividade (18 a 64 anos), aproximadamente 53,3\% estão aposentados, mesmo que $73,3 \%$ tenham apresentado independência completa na MIF com média de 108,93 $( \pm 11,38)$ e moda de 115 , valores compatíveis com o desempenho competente de papéis ocupacionais considerados comuns na referida faixa etária.

Os níveis de dependência moderada e de independência, respectivamente, relacionados com o desempenho dos papéis ocupacionais, encontram-se nas tabelas 1 e 2, dando-se destaque aos resultados encontrados para os papéis de estudante, trabalhador, e serviço doméstico. Observa-se que no presente muitos sofreram ruptura em seu desempenho, e muitos sujeitos não têm perspectiva de resgatar estes papéis, além de apresentarem alguns ausentes ao longo da vida.

Para essa amostra não foram encontradas rela-

Tabela 1 - Relação entre o número de sujeitos que apresentam dependência moderada com o desempenho de papéis ocupacionais, em porcentagem. Curitiba, 2009

\begin{tabular}{lccccccccc}
\hline & $\mathbf{1}$ & $\mathbf{2}$ & $\mathbf{3}$ & $\mathbf{4}$ & $\mathbf{5}$ & $\mathbf{6}$ & $\mathbf{7}$ & $\mathbf{8}$ & $\begin{array}{c}\text { Total } \\
\mathbf{( \% )}\end{array}$ \\
\hline Estudante & 50 & 30 & - & - & - & - & 10 & 10 & 100 \\
Trabalhador & 30 & 40 & - & - & - & 10 & 10 & 10 & 100 \\
Voluntário & - & 10 & - & - & - & 50 & - & 40 & 100 \\
Cuidador & 10 & 40 & - & - & - & 20 & 10 & 20 & 100 \\
Serviço Doméstico & - & 40 & - & - & - & 10 & 50 & - & 100 \\
Amigo & - & 20 & - & - & - & 10 & 40 & 30 & 100 \\
Membro de Família & - & 10 & - & - & - & - & 90 & - & 100 \\
Religioso & 20 & 30 & - & - & - & 10 & 10 & 30 & 100 \\
Passatempo/Amador & - & 20 & - & 10 & 10 & - & 60 & - & 100 \\
Organizações & 10 & 20 & - & - & - & 20 & 10 & 40 & 100 \\
\hline
\end{tabular}

1 - Desempenho somente no passado; 2 - Perda somente no presente; 3 - Ganho somente no presente; 4 - Desempenho a partir do presente; 5 - Desempenho até o presente; 6 - Novo papel no futuro; 7 - Papel contínuo; 8 - Papel ausente.

ções relevantes entre o tempo de doença, o nível de independência funcional e o desempenho de papéis, nem entre o nível de educação formal e as demais variáveis. Todavia, não é possível afirmar ou refutar a inexistência dessas relações. Para tanto, seria necessária a realização de estudos com orientação prospectiva para acompanhamento temporal dos sujeitos e concomitante análise da evolução de seus níveis de independência, do desempenho de papéis, e dos fatores envolvidos nessas mudanças. 
Tabela 2 - Relação entre o número de sujeitos que apresentam independência com o desempenho de papéis ocupacionais, em porcentagem. Curitiba, 2009

\begin{tabular}{lccccccccc}
\hline & $\mathbf{1}$ & $\mathbf{2}$ & $\mathbf{3}$ & $\mathbf{4}$ & $\mathbf{5}$ & $\mathbf{6}$ & $\mathbf{7}$ & $\mathbf{8}$ & $\begin{array}{c}\text { Total } \\
\mathbf{( \% )}\end{array}$ \\
\hline Estudante & 61 & 28 & - & - & 03 & 03 & 06 & - & 100 \\
Trabalhador & 22 & 39 & - & 06 & 03 & 06 & 22 & 03 & 100 \\
Voluntário & 06 & 17 & 03 & - & - & 50 & 08 & 17 & 100 \\
Cuidador & 11 & 17 & - & 06 & - & 19 & 42 & 06 & 100 \\
Serviço Doméstico & - & 03 & - & 03 & 03 & 03 & 89 & - & 100 \\
Amigo & 06 & 31 & - & 03 & - & 11 & 36 & 14 & 100 \\
Membro de Família & 03 & 06 & - & - & 06 & 03 & 75 & 08 & 100 \\
Religioso & 17 & 25 & - & 03 & - & 22 & 08 & 25 & 100 \\
Passatempo/Amador & 03 & 03 & - & 11 & 03 & 03 & 78 & - & 100 \\
Organizações & 03 & 17 & - & - & - & 17 & 06 & 58 & 100 \\
\hline
\end{tabular}

1 - Desempenho somente no passado; 2 - Perda somente no presente; 3 - Ganho somente no presente; 4 - Desempenho a partir do presente; 5 - Desempenho até o presente; 6 - Novo papel no futuro; 7 - Papel contínuo; 8 - Papel ausente.

\section{DISCUSSÃO}

De modo geral, observa-se que os participantes desta pesquisa apresentaram altos índices de ruptura no desempenho dos papéis no presente, sem perspectiva de resgatá-los no futuro. Duas contradições foram destacadas nesse âmbito: os baixos índices de desempenho dos papéis foram contrastados com a alta importância atribuída a estes, e principalmente com os níveis de independência funcional compatíveis com a realização dos mesmos - em especial o de trabalhador.

Com base nisso, nota-se que a condição de saúde, ou doença, e as deficiências decorrentes dessas, podem não ser os principais fatores interferentes no desempenho de sujeitos que apresentam sequelas neurológicas, uma vez que a grande maioria apresentou independência funcional e, mesmo assim, ruptura de papéis ocupacionais.

De acordo com Modelo da Ocupação Humana, as ocupações, e consequentemente os papéis ocupacionais, são assumidos pelos sujeitos a partir de uma relação dinâmica entre sua capacidade de desempenho, sua habilidade de desenvolver e adotar hábitos, e sua motivação para assumir essas ocupações, seus interesses, valores e seu senso de efetividade no contexto onde está inserido. Todos esses aspectos influenciam e são influenciados também por esse contexto, pelo ambiente físico, social, temporal e cultural no qual o sujeito se encontra ${ }^{(5)}$. Em suma, o engajamento, a manutenção, e a perda de papéis ocupacionais são influenciados por outros fatores que não apenas as condições de saúde/ doença ou deficiências como as apresentadas por sujeitos com sequelas neurológicas.

Essa perspectiva corrobora com a abordagem biopsicossocial proposta pela Organização Mundial da Saúde (OMS) e apresentada na Classificação Internacional de Funcionalidade, Incapacidade e Saúde(CIF) (8). Conforme essa abordagem, o desempenho de atividades e a participação dos participantes avaliados sofrem influência tanto de suas condições de saúde/ doença, consequentes deficiências, quanto de fatores pessoais e ambientais, como apresentado na figura 1.

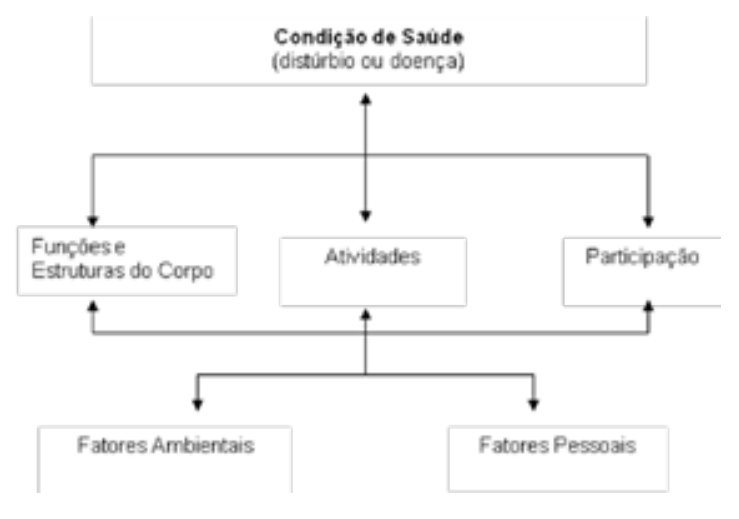

Cogitare Enferm. 2013 Out/Dez; 18(4):676-81 


\section{CONSIDERAÇÕES FINAIS}

Por meio do protocolo de avaliação da Medida de Independência Funcional e da Lista de Identificação de Papéis Ocupacionais foi possível observar que, apesar do nível de independência completa ou dependência moderada, e do alto grau de importância designada aos papéis ocupacionais, as pessoas sequelas neurológicas incluídos nesta pesquisa apresentaram um padrão de ruptura no desempenho de seus papéis ocupacionais, especialmente em relação ao papel de trabalhador.

A aquisição de aposentadoria e/ou benefícios em idade ainda considerada produtiva pôde ser apontada como um dos fatores marcantes do rompimento no desempenho de papéis, principalmente de estudante e trabalhador.

Frente aos dados encontrados neste estudo, notase que a condição de saúde e a deficiência podem não ser os principais fatores interferentes no desempenho ocupacional dos sujeitos que apresentam sequelas neurológicas. Essas condições se associam a um conjunto de fatores pessoais e ambientais presentes no contexto onde o sujeito está inserido.

Pesquisas futuras, prospectivas, poderão dar maior clareza sobre quais os fatores interferentes na ruptura e retorno do sujeito aos seus papéis ocupacionais, de que forma essa dinâmica ocorre, e como tais fatores se relacionam entre si.

\section{REFERÊNCIAS}

1. Faria I. Neurologia adulto: disfunções neurológicas. In: Cavalcanti A, Galvão C. Terapia Ocupacional: fundamentação e prática. Rio de Janeiro: Guanabara Koogan; 2007. p. 187- 204.

2. Nickel R, Lima AP, Navarro EJ, Pinto LM, Teive HAG, Becker, N. Correlação entre a qualidade de vida de cuidadores familiares e os níveis de independência funcional dos cuidados. Cogitare enferm. 2010;15(2):225-30.

3. Riberto M, Miyazaki MH, Jucá SSH, Lourenço C, Battistella LR. Independência funcional em pessoas com lesões encefálicas adquiridas sob reabilitação ambulatorial. Acta Fisiátr. 2007;14(2):87-94.

4. Oliveira LMB. Cartilha do Censo de 2010 - Pessoas com Deficiência/Secretaria de Direitos Humanos da Presidência da República (SDH/PR) / Secretaria Nacional de Promoção dos Direitos da Pessoa com Deficiência (SNPD) / Coordenação-Geral do Sistema de Informações sobre a Pessoa com Deficiência; Brasília: SDH-PR/SNDP, 2012.
4. Kielhofner G. Model of human occupation: theory and application. $4^{\mathrm{a}}$ ed. Philadelphia: the Point; 2008.

5. Riberto M, Miyazaki MH, Jucá SSH, Sakamoto H, Pinto PPN, Battistella RL. Validação da versão brasileira da medida de independência funcional. Acta Fisiátr. 2004;11(2):72-6.

6. Cordeiro JJR. Validação da lista de identificação de papéis ocupacionais em pacientes portadores de doença pulmonar obstrutiva crônica (DPOC) no Brasil [dissertação]. São Paulo (SP): Universidade Federal de São Paulo; 2005.

7. Organização Mundial da Saúde (OMS). CIF: Classificação internacional de funcionalidade, incapacidade e saúde. São Paulo: Edusp; 2003.

8. Freitas WD. O acesso à educação das pessoas com deficiência: uma análise da exclusão de crianças e adolescentes beneficiárias do benefício de prestação continuada da assistência social - BPC [dissertação]. Rio de Janeiro (RJ): Fundação Oswaldo Cruz; 2010.

9. Spencer JC. Avaliação dos contextos de desempenho. In: Neistadt E, Crepeau EB, organizadores. Willard \& Spackman Terapia Ocupacional. $9^{\mathrm{a}}$ ed. Rio de Janeiro: Guanabara Koogan; 2002. p. 266-83. 